Article

\title{
The AGPase Family Proteins in Banana: Genome-Wide Identification, Phylogeny, and Expression Analyses Reveal Their Involvement in the Development, Ripening, and Abiotic/Biotic Stress Responses
}

\author{
Hongxia Miao ${ }^{1,+}$, Peiguang Sun ${ }^{2,+}$, Qing Liu ${ }^{3, \dagger}$, Juhua Liu ${ }^{1}$, Biyu $\mathrm{Xu}^{1, *}$ and Zhiqiang Jin ${ }^{1,2, *}$ \\ 1 Key Laboratory of Tropical Crop Biotechnology, Ministry of Agriculture, \\ Institute of Tropical Bioscience and Biotechnology, Chinese Academy of Tropical Agricultural Sciences, \\ Haikou 571101, China; miaohongxia@itbb.org.cn (H.M.); liujuhua@itbb.org.cn (J.L.) \\ 2 Key Laboratory of Genetic Improvement of Bananas, Hainan Province, Haikou Experimental Station, \\ Chinese Academy of Tropical Agricultural Sciences, Haikou 570102, China; sunpeiguang@catas.cn \\ 3 Commonwealth Scientific and Industrial Research Organization Agriculture and Food, Canberra, \\ ACT 2601, Australia; Qing.Liu@csiro.au \\ * Correspondence: xubiyu@itbb.org.cn (B.X.); jinzhiqiang@itbb.org.cn (Z.J.); \\ Tel.: +86-898-6696-0172 (B.X.); Fax: +86-898-6689-0978 (B.X.) \\ + These authors contributed equally to this work.
}

Received: 29 June 2017; Accepted: 18 July 2017; Published: 25 July 2017

\begin{abstract}
ADP-glucose pyrophosphorylase (AGPase) is the first rate-limiting enzyme in starch biosynthesis and plays crucial roles in multiple biological processes. Despite its importance, AGPase is poorly studied in starchy fruit crop banana (Musa acuminata L.). In this study, eight MaAGPase genes have been identified genome-wide in $M$. acuminata, which could be clustered into the large (APL) and small (APS) subunits. Comprehensive transcriptomic analysis revealed temporal and spatial expression variations of MaAPLs and MaAPSs and their differential responses to abiotic/biotic stresses in two banana genotypes, Fen Jiao (FJ) and BaXi Jiao (BX). MaAPS1 showed generally high expression at various developmental and ripening stages and in response to abiotic/biotic stresses in both genotypes. MaAPL-3 and - $2 a$ were specifically induced by abiotic stresses including cold, salt, and drought, as well as by fungal infection in FJ, but not in BX. The presence of hormone-related and stress-relevant cis-acting elements in the promoters of MaAGPase genes suggests that MaAGPases may play an important role in multiple biological processes. Taken together, this study provides new insights into the complex transcriptional regulation of AGPases, underlying their key roles in promoting starch biosynthesis and enhancing stress tolerance in banana.
\end{abstract}

Keywords: banana (Musa acuminata L.); AGPase; genome-wide identification; fruit development; abiotic stress; biotic stress

\section{Introduction}

Starch is the most abundant storage polysaccharide in plants. As an important carbohydrate and energy source in the human diet and nutrition, starch is massively produced in cassava (Manihot esculenta) roots [1], potato (Solanum tuberosum) tubers [2], cereal seeds [3], and a number of fresh fruits such as banana (Musa acuminata) [4-8]. The biosynthesis of starch in plants is a complex process involving a variety of enzymes, such as ADP-glucose pyrophosphorylase (AGPase), granule-bound starch synthase (GBSS), soluble starch synthase (SS), starch branching enzyme (SBE), and debranching enzyme (DBE) $[2,9,10]$. AGPase, as the first key regulatory and rate-limiting enzyme 
in starch biosynthetic pathways, controls the starch content and affects the yield and quality of plants [2,9-12].

AGPase catalyzes the synthesis of the activated glycosyl donor and ADP-glucose from Glucose-1-phosphate (Glc-1-P) and ATP. It is a heterotetrameric enzyme that is composed of two large (APL) and two small (APS) subunits with typical NTP transferases domains (PF00483) [2,13]. The APL has a regulatory function with defective catalytic properties in S. tuberosum, Arabidopsis (Arabidopsis thaliana), and barley (Hordeum vulgare) [14]. In contrast, the APS is regulatory-deficient but it plays more of a catalytic role in plant tissues [15]. To date, genome-wide analysis has identified a variable number of APL and APS in numerous higher plants, including six in A. thaliana (four APLs and two APSs) [15], seven in rice (Oryza sativa) (four APLs and three APSs) [16,17], six in maize (Zea mays) (four APLs and two APSs) [13,18], six in sweet potato (Ipomoea batatas) (four APLs and two APSs) [19], four in sago palm (Metroxylon sagu) (three APLs and one APS) [20], four in kiwifruit (Actinidia deliciosa) (three APLs and one APS) [21], and four in tomato (Solanum lycopersicon) (three APLs and one APS) [22].

Further, recent biochemical and functional analyses have revealed that AGPase is playing an active role in multiple plant biological processes, including growth, development, environmental adaptation, and host-pathogen interactions. For example, in A. thaliana, AtAGPase was found to regulate plantlet growth rate and root development [23,24]. A tobacco (Nicotiana tabacum) NtAGPase was found to play a crucial role in petal expansion and growth [25]. The regulated expression of an APS derived from cotton (Gossypium hirsutum), together with the presence of starch in cotton fiber suggested an important role of GhAGPase in fiber development [26]. Temporally extended expression of APL1 enhanced accumulation of starch and soluble solids in mature fruit in S. lycopersicon [22]. A mutant defective in OsAPL4 showed impaired starch biosynthesis in pollen grains, which led to male sterility in O. sativa [27]. Furthermore, the expression of numerous AGPase genes in plants was found to be regulated by various stress conditions, including cold, salt, drought, and plant-pathogens [12,28-30]. In S. tuberosum, the expression of AGPase was regulated by low storage temperatures [30]. Under salt stress, APL1 from S. lycopersicum was up-regulated in developing fruits, suggesting its role in the promotion of starch biosynthesis under the salinity stress in ABA- and osmotic stress-independent manners [28]. Under drought stress, AGPase activity was inhibited leading to significant losses in crop yield [12,31,32]. The Downy mildew (Plasmopara viticola) infection was shown to significantly induce AGPase activity and abnormal starch accumulation in grapevine leaves [29]. Taken together, these studies have revealed many important roles of the AGPase gene family in the regulation of plant growth, development, and responses to abiotic/biotic stresses.

With starch content reaching $74-88 \%$ of its dry weight in unripe fruit, M. acuminata is not only the most popular starch-rich fresh fruit in the world, but also an important staple food in some African and Latin American countries [6-8,33,34]. Starch biosynthesis is of crucial importance since starch content determines fruit yield, quality, and economic value of banana. As a large annual monocotyledonous herbaceous plant, banana is frequently affected or even destroyed by various abiotic/biotic stresses during growth and development. Genome-wide investigations of key genes involved in fruit development and abiotic/biotic stress responses have been conducted in several fruit crops, including banana [35-37]. The involvement of AGPase in the ripening of banana fruit has recently reported [5]. However, a genome-wide investigation of the AGPase gene family leading to an integrated picture of the potential functionality of this important rate-limiting enzyme involved in starch biosynthesis in banana is still lacking.

In this study, we identified eight MaAGPase genes from the entire banana genome and conducted a range of molecular analyses on their phylogenetic relationship, gene structure, protein motifs, spatial and temporal expression patterns, and expression in response to different abiotic/biotic stresses (cold, salt, drought, and fungal infection) in two banana varieties. In addition, we analyzed in silico the hormone-related and stress-relevant cis-elements in the promoters of MaAGPase genes. This comprehensive study serves to facilitate our understanding of MaAGPase in association with 
fruit development processes and abiotic/biotic stress responses, and provides a foundation for future studies of crop improvement involving AGPases.

\section{Results}

\subsection{Identification and Phylogenetic Analysis of Banana MaAGPase Genes}

DNA sequence database search using BLAST and the hidden Markov models (HMM) was conducted to identify all banana MaAGPase genes using the typical NTP transferase domains (PF00483), A. thaliana AtAGPase and O. sativa OsAGPase sequences as queries. A total of eight MaAGPase genes were identified in the M. acuminata genome and designated as MaAPL-1a, $-1 b,-2 a,-2 b,-2 c,-3$, MaAPS-1, and -2 following the nomenclature of their respective orthologous genes in $A$. thaliana. The eight predicted MaAGPase proteins varied from 450 (MaAPS2) to 549 (MaAPL2a) amino acid residues in size, with relative molecular masses between 50.580 (MaAPS2) and 60.699 (MaAPL2a) kDa, and isoelectric points ranging from 6.53 to 8.91 (Figure S1 and Table S1).

To study the evolutionary relationships between AGPase family proteins, a neighbor-joining tree was generated by aligning eight, six, and seven AGPase proteins from M. acuminata, A. thaliana, and O. sativa, respectively, using ClustalX and MEGA5.0 software. As shown in Figure 1, MaAGPase proteins were clearly grouped based on the size of their subunits. Six M. acuminata APL genes (MaAPL-1a, -1b, -2a, -2b, -2c, and -3) together with A. thaliana AtAPL-1, -2, -3, -4, and O. sativa OsAPL-1, $-2,-3,-4$ were classified into the large subunit group, while the other two MaAGPase genes, i.e., MaAPS-1 and -2 together with AtAPS-1, -2 , OsAPS-1, $-2 a$, and $-2 b$, were classified into the small subunit group.

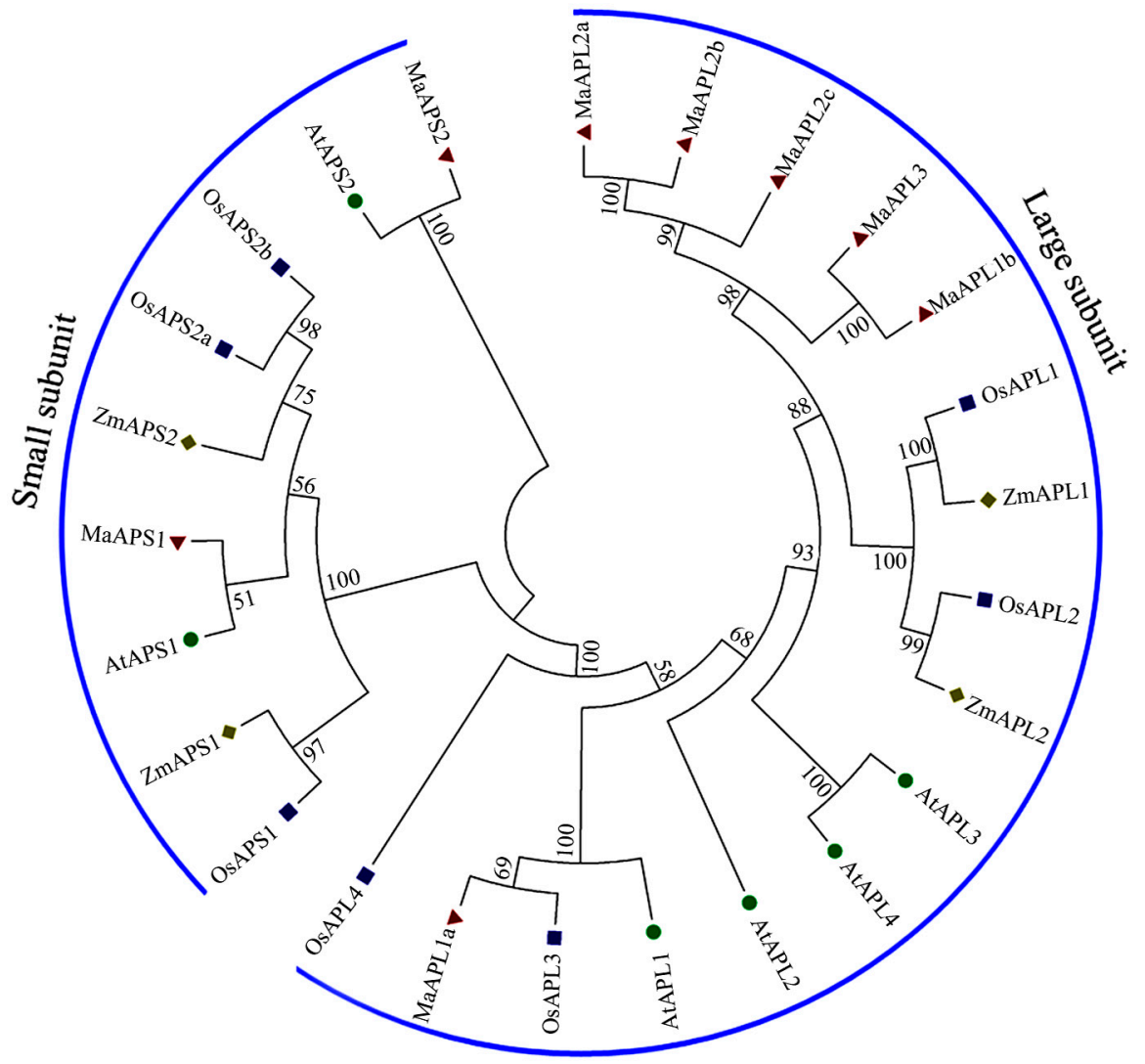

Figure 1. Phylogenetic analysis of the AGPases from Arabidopsis, rice, and banana. The Neighbor-joining tree was drawn using MEGA5.0 with 1000 bootstraps. Two subgroups were identified and classified as large subunit and small subunit. The circle, square, and triangle represent AGPase proteins from Arabidopsis, rice, and banana, respectively. 


\subsection{Gene Structure and Conserved Motif Analysis of Banana MaAGPase Genes}

Evolutionary analysis was further supported by the exon-intron structural divergence within families (Figure 2). The gene organization and structural diversity of MaAGPase genes were analyzed by comparing to their orthologous genes derived from A. thaliana, O. sativa, and Z. mays using Gene Structure Display Server (GSDS) web based software. The gene organization was different between $A P L$ and APS genes in all the four species, indicating the diversity of expansion and evolution between these two subunit groups in plant AGPase genes (Figure 2).

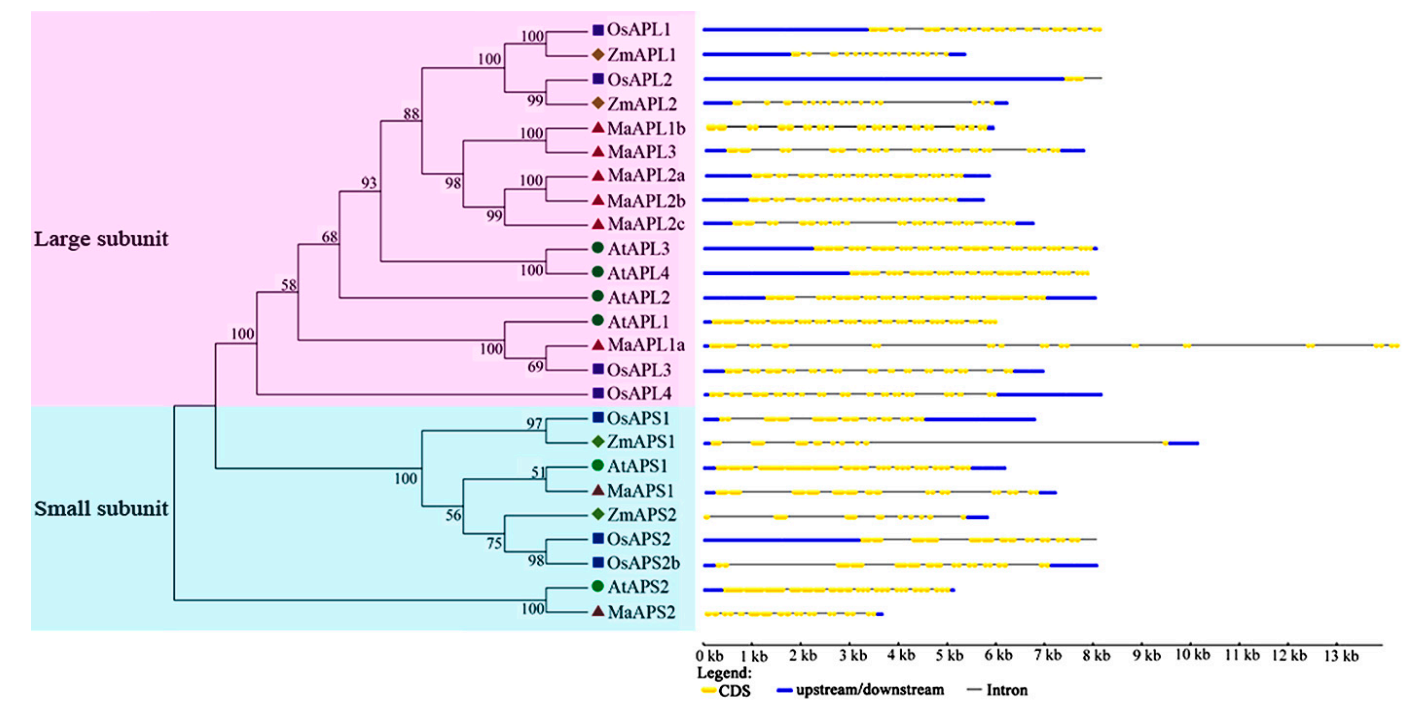

Figure 2. Gene structure analyses of MaAGPases. Exon-intron structure analyses were performed using the Gene Structure Display Server database. Blue boxes indicate upstream/downstream; yellow boxes indicate exons; black lines indicate introns. The circle, square, diamond, and triangle represent AGPase proteins from Arabidopsis, rice, maize, and banana, respectively.

To further explore the protein structural diversity and predict the functionality of MaAGPase gene family, a total of 15 conserved motifs in MaAGPase proteins were identified using MEME5.2 software and annotated with the InterPro database (Figure 3, Table S2). Four motifs (motifs 1, 5-7) were annotated as NTP transferases (PF00483), which is characteristic of the AGPase protein family. In specific, six MaAPL proteins contain the motifs 1-15 whereas all MaAPS proteins contain motifs $2-8,12$, and 13 . It is probable that the motif structure conservation and variation reflect the specific evolutionary history and functionality divergence among the eight MaAGPases.

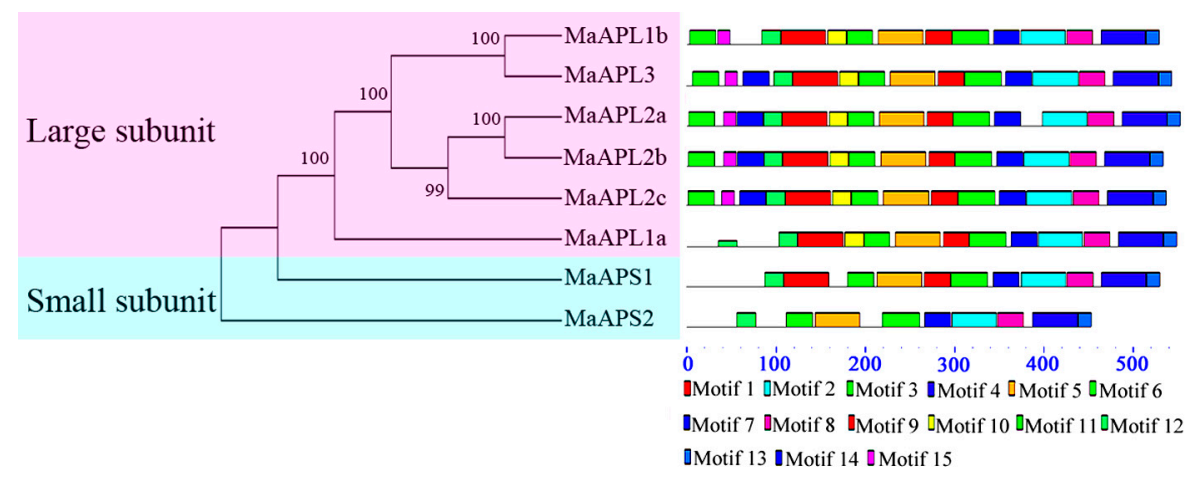

Figure 3. Phylogenetic and motif analyses of MaAGPase proteins. All proteins were identified by MEME database with the complete amino acid sequences of each MaAGPase identified. MaAGPases were classified into large and small subunits based on their phylogenetic relationship. 


\subsection{Spatial Expression Analysis of MaAGPase Genes in Banana}

To investigate the spatial expression patterns of MaAGPase genes and their potential functional roles in banana growth and development, roots, leaves, and fruits were sampled from both BX and FJ genotypes and subjected to transcriptional analysis. Five MaAGPase genes were found to express in at least one of the tested tissues in both genotypes (Figure 4, Table S3). However, the expression of MaAPL-1a, $-2 b$, and MaAPS2 was not detected.

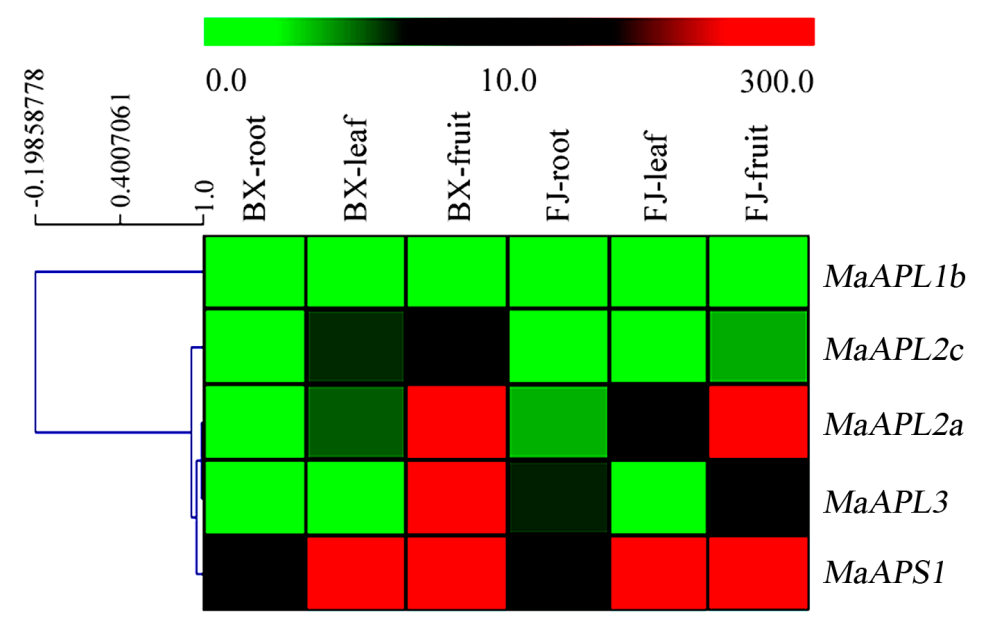

Figure 4. Expression of MaAGPases in roots, leaves, and fruits of BX and FJ banana varieties. The heat map with clustering was created based on the FPKM value of the MaAGPases. Differences in gene expression changes are shown in color in the green-red scale.

In BX, MaAPS1 showed transcriptional expression in all tissue types examined. However, its highest expression was detected in the fruits with FPKM (fragments per kilobase of exon per million fragments, FPKM) > 10, together with other MaAGPase members including MaAPL-2a, $-2 c$, and -3 .

In FJ, five, four, and five MaAGPases were expressed in roots, leaves, and fruit, respectively. MaAPS1 was highly expressed in all the tissues examined (FRKM > 10). High expression levels of MaAPL-2 $a$ and -3 were also detected in fruits.

In the three tissues examined, consistent spatial expression patterns of $M a A P L-1 b,-2 a,-3$, and MaAPS1 were observed between the two genotypes, BX and FJ. MaAPS1 exhibited consistently high level of expression (FRKM > 40) in all tissues examined in both genotypes, suggesting its important and divergent functional role in banana growth and development. This is in sharp contrast to the expression of MaAPL2c in fruit, which showed high level expression (FRKM > 47) in BX, but low expression $(F R K M<4)$ in FJ. This may suggest a differential functional role of this gene during fruit development in divergent banana cultivars. Taken together, this genotype-based spatial expression analysis warrants further study on the functional roles of MaAGPase gene family in banana.

\subsection{Temporal Expression Analysis of MaAGPase Genes during Banana Fruit Development and Ripening}

To investigate the potential functionality of MaAGPase genes during banana fruit development and postharvest ripening, the expression of MaAGPases was analyzed in fruits sampled 0, 20, and 80 day after emergence from pseudostem (DAF) in both BX and FJ genotypes, 8 and 14 day after harvest (DPH) from BX, 3 and 6 DPH from FJ (Figure 5, Table S4). Except MaAPL-1a, -2b, and MaAPS2, five MaAGPase genes were expressed during fruit development and postharvest ripening in these two genotypes. 


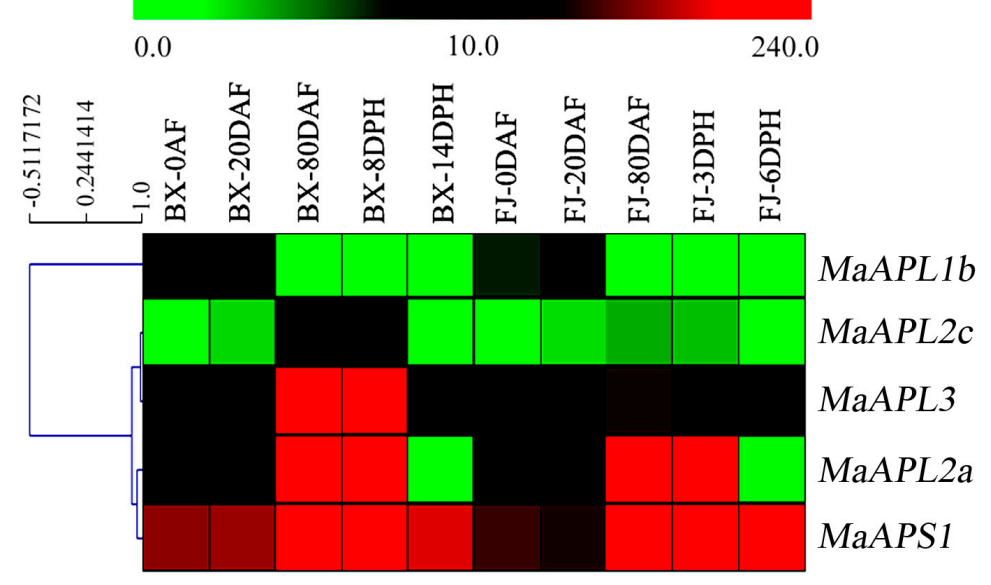

Figure 5. Expression of MaAGPases during different stages of fruit development and ripening in two banana varieties, BX and FJ. The heat map with clustering was created based on the FPKM value of the MaAGPases. Differences in gene expression changes are shown in color in the green-red scale.

In BX, notably, MaAPS1 showed the highest transcriptional accumulation (FRKM > 126) at all the examined stages of fruit development and ripening. Four other MaAGPase genes, including MaAPL-1b, $-2 a,-2 c$, and -3 were also expressed at all the stages examined, but at relatively lower level than MaAPS1. Further, three (MaAPL-1b, $-2 a$, and -3), three (MaAPL-1b, $-2 a$, and -3), three (MaAPL-2a, $-2 c$, and -3), three (MaAPL-2a, -2c, and -3) MaAGPase genes were detected in high transcriptional abundance (FRKM > 10) at 0,20, $80 \mathrm{DAF}$, and $8 \mathrm{DPH}$, respectively. Similar to BX, MaAPS1 exhibited high expression (FPKM $>76$ ) at all the examined stages of fruit development and ripening in FJ. Four other MaAGPase genes, including MaAPL-1b,-2a,-2c, and -3 were also expressed, among which two (MaAPL-2a and -3), three (MaAPL-1b,-2a, and -3), two (MaAPL-2a and -3), two (MaAPL-2a and -3), and one (MaAPL3) genes showed high transcription level (FPKM $>10)$ at $0,20,80 \mathrm{DAF}$, and 3 and $6 \mathrm{DPH}$, respectively. During fruit development and ripening, the two large subunit genes (MaAPL-3 and $-2 a$ ) showed similar expression patterns in both $\mathrm{BX}$ and FJ. In contrast, another large subunit gene, MaAPL2c, showed much higher expression level (FPKM > 29) in BX, compared to FJ $($ FPKM < 3.9) at $80 \mathrm{DAF}$ and $8 \mathrm{DPH}$. These findings imply a significant transcriptional response of MaAPL2c during fruit development and early-stage ripening processes in BX. In addition, MaAPS1 displayed high transcriptional abundance (FPKM > 76) at all phases in both BX and FJ, implying the high expression of small subunit gene MaAPS1 could be important in the regulation of fruit development and ripening.

\subsection{Expression of MaAGPases under Cold, Salt, and Osmotic Stresses}

MaAGPase expression in response to cold, salt, and osmotic treatments was analyzed by transcriptome data in $\mathrm{BX}$ and FJ. Five out of the eight MaAGPase genes showed variable levels of transcription in banana leaves following the abiotic stress treatments (Figure 6, Table S5).

In BX, the expression of MaAPS1 was strongly up-regulated (FPKM > 134) by all the three abiotic stress treatments. The two large subunit genes, MaAPL-2a and $-2 c$ were only moderately up-regulated by cold and salt treatments (FPKM $>2.0$ ). A similar level of up-regulation by osmotic treatment was also observed in MaAPL2a, but not in MaAPL-2c. MaAPL-1 $b$ and -3 were all down-regulated by any of the three abiotic treatments (FPKM < 0.5). MaAPL2c was also down-regulated by osmotic treatment, but not by cold and salt treatments. Similar to BX, the expression of MaAPS1 was significantly induced (FPKM > 329) in FJ by cold, salt, and osmotic treatments. In FJ, moderate inductions were also observed in the three large subunit genes, including $M a A P L-2 a,-2 c$, and -3 (FPKM $>2.5$ ) under each of the three abiotic stress treatments. The expression of MaAPL1b in FJ was down-regulated (FPKM $<0.06$ ) following each of the three abiotic stress treatments, consistent with the observation in BX. MaAPL3, was specifically up-regulated $(\mathrm{FPKM}>2.6)$ in FJ, but maintained low expression in BX $(\mathrm{FPKM}<0.19)$ 
under either of the three stress treatments, implying a significant genotypic variation in response to abiotic treatments.

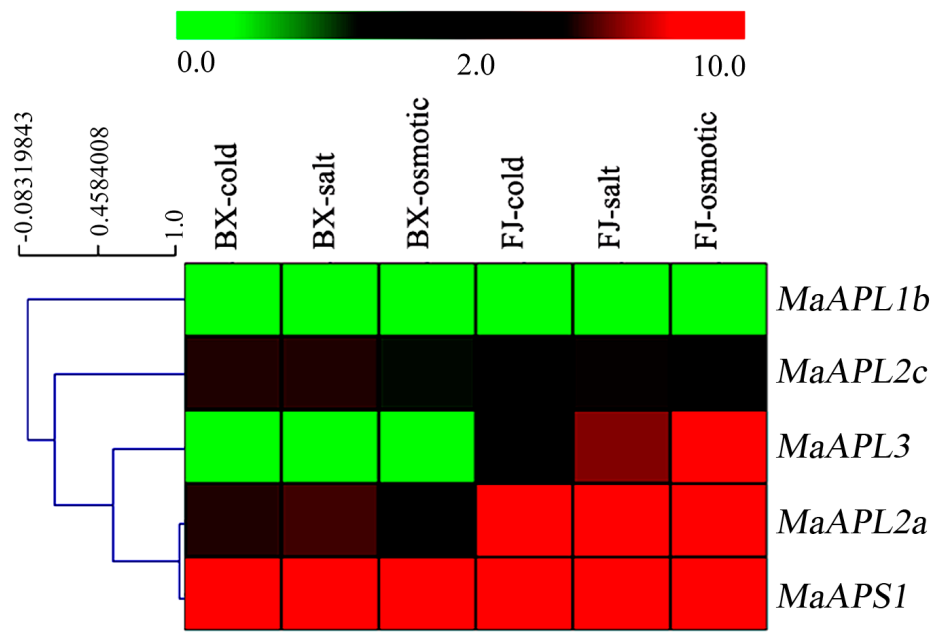

Figure 6. Expression of MaAGPases in response to cold, salt, and osmotic stresses in two banana varieties, BX and FJ. The heat map with clustering was created based on the FPKM value of the MaAGPases. Differences in gene expression are shown in color in the green-red scale.

\subsection{Expression Profiles of Banana MaAGPases in Response to Fusarium Oxysporum f.sp. Cubense (Foc)} Tropical Race 4 (TR4) Infection

To investigate the potential functional roles of MaAGPase genes in defense against fungal diseases in banana, MaAGPase gene expression was analyzed in the entire root system of $\mathrm{BX}$ and FJ plants following 0 and 2 days post-infection (DPI) with the Panama disease, Foc TR4. Five out of the eight MaAGPase genes showed transcriptional changes in response to the fungal infection in both $\mathrm{BX}$ and FJ genotypes (Figure 7 and Table S6).

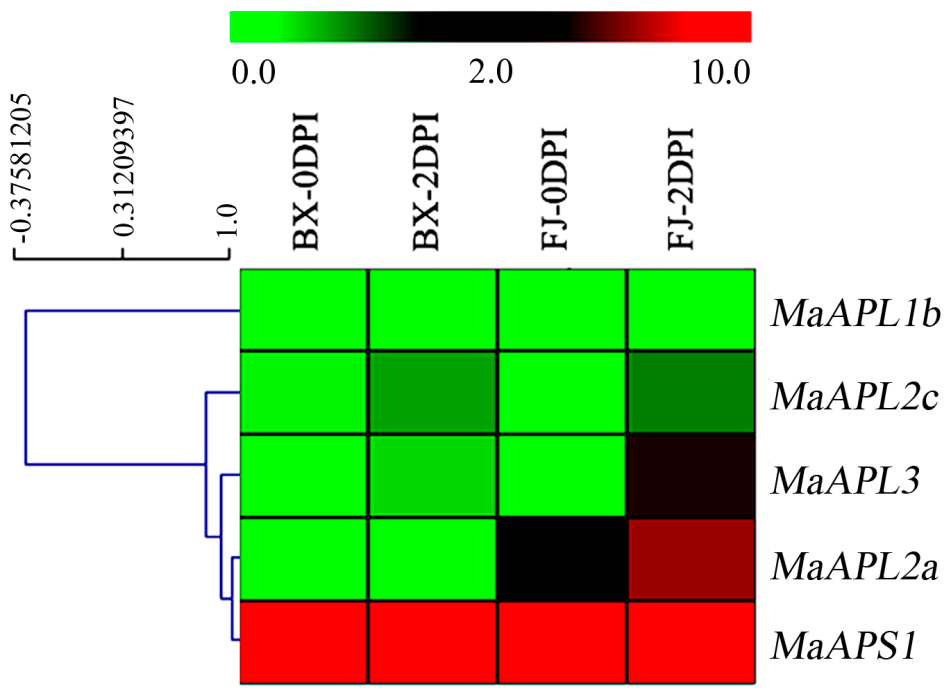

Figure 7. Expression of MaAGPases in response to fungal disease in two banana varieties, BX and FJ. The heat map with clustering was created based on the FPKM value of the MaAGPases. Differences in gene expression changes are shown in color in green-red scale. 
In both BX and FJ, five MaAGPase genes, including MaAPL-1b, $-2 a,-2 c,-3$, and MaAPS1, were expressed in Foc TR4 infected roots. In BX, MaAPS1 exhibited a 1.6-fold increase in sequence abundance at 2 DPI. In FJ, MaAPL-2a, -3, and MaAPS1 were all up-regulated, with 46-, 3.5-, and 1.6-fold increase, respectively.

2.7. Validation of the Differentially Expressed MaAGPase Genes by Quantitative Real-Time Polymerase Chain Reaction (qRT-PCR) Analysis

RNA-seq analysis indicated that MaAPL-2a, -3, and MaAPS1 had exhibited high levels of expression in different tissues, or during most stages of fruit development and ripening, or being induced by abiotic/biotic stress treatments in BX or FJ. Such a feature of these three genes was verified by qRT-PCR analysis. After normalization, all the examined MaAGPases with the exception of MaAPL3 in FJ at 6DPH and MaAPS1 in BX at 8DPH showed consistent expression patterns with the RNA-seq analysis (Figure 8). The correlation coefficient between RNA-seq and qRT-PCR data ranged from 0.8750 to 0.9999 (Table S7). These results indicate that RNA-seq analysis supplied suitable expression results for both banana varieties.

\subsection{In Silico Identification of Hormone-Related and Stress-Related Cis-Acting Elements in the Promoters of MaAGPase Genes}

Promoter is a molecular switch that initiates gene expression and studies of the cis-acting elements in promoter sequence have been used as a useful tool for investigating regulatory mechanism of gene expression and gene function [10]. As shown in Table 1, five hormone-related (ABA, auxin, MeJA, ethylene, and gibberellin) and seven abiotic or biotic stress-related (anaerobic, fungal, heat, cold, drought, salicylic acid, and defense) elements were identified in the promoters of these MaAGPase genes. No less than two hormone- or stress-related elements were found in each of the eight MaAGPase promoters, suggesting the potentially important roles of AGPases in the regulation of development, ripening, and stress responses through the hormone- and stress-related cis-acting elements. 
Table 1. Kinds and numbers of the known hormone-related and stress-related elements found in the upstream regions of MaAGPase genes.

\begin{tabular}{|c|c|c|c|c|c|c|c|c|c|c|c|c|c|c|}
\hline Element & $\begin{array}{l}A B R E \\
\text { (ABA) }\end{array}$ & $\begin{array}{c}A R E \\
\text { (Anaerobic) }\end{array}$ & $\begin{array}{l}\text { AuxRR } \\
\text { (Auxin) }\end{array}$ & $\begin{array}{l}\text { Box-W1 } \\
\text { (Fungal) }\end{array}$ & $\begin{array}{l}\text { CGTCA-Motif } \\
\text { (MeJA) }\end{array}$ & Circadian & $\begin{array}{c}E R E \\
\text { (Ethylene) }\end{array}$ & $\begin{array}{c}\text { GARE } \\
\text { (Gibberellin) }\end{array}$ & $\begin{array}{c}\text { HSE } \\
\text { (Heat) }\end{array}$ & $\begin{array}{l}\text { LTR } \\
\text { (Cold) }\end{array}$ & $\begin{array}{l}M B S \\
\text { (Drought) }\end{array}$ & $\begin{array}{l}\text { TCA-Element } \\
\text { (Salicylic } \\
\text { Acid) }\end{array}$ & $\begin{array}{c}\text { TC-Rich } \\
\text { Repeats } \\
\text { (Defense) }\end{array}$ & Total \\
\hline MaAPL1a & 0 & 2 & 0 & 0 & 0 & 1 & 0 & 0 & 0 & 0 & 4 & 0 & 0 & 7 \\
\hline MaAPL1b & 0 & 2 & 0 & 1 & 3 & 1 & 0 & 0 & 0 & 0 & 1 & 0 & 2 & 10 \\
\hline MaAPL2a & 3 & 0 & 1 & 1 & 5 & 2 & 0 & 0 & 0 & 0 & 1 & 0 & 3 & 16 \\
\hline MaAPL2b & 3 & 0 & 1 & 1 & 4 & 2 & 0 & 0 & 0 & 0 & 1 & 2 & 3 & 17 \\
\hline MaAPL2c & 4 & 1 & 0 & 0 & 1 & 0 & 1 & 1 & 0 & 2 & 0 & 1 & 1 & 12 \\
\hline MaAPL3 & 4 & 0 & 1 & 0 & 7 & 1 & 0 & 1 & 0 & 0 & 1 & 0 & 0 & 15 \\
\hline MaAPS1 & 2 & 0 & 0 & 0 & 0 & 1 & 0 & 1 & 0 & 0 & 3 & 0 & 0 & 6 \\
\hline MaAPS2 & 0 & 0 & 0 & 0 & 0 & 0 & 0 & 0 & 2 & 0 & 0 & 0 & 0 & 2 \\
\hline
\end{tabular}

ABRE, ABA responsive element; $A R E$, anaerobic responsive element; AuxRR, auxin responsive element; Box-W1, fungal elicitor responsive element; CGTCA-motif, MeJA responsive element; Circadian, circadian control; ERE, ethylene responsive element; GARE, gibberellins responsive element; HSE, heat stress responsive element; $L T R$, low temperature responsive element; MBS, MYB binding site involved in drought induction; TCA-element, salicylic acid responsive element; TC-rich repeats, defense responsive elements. 

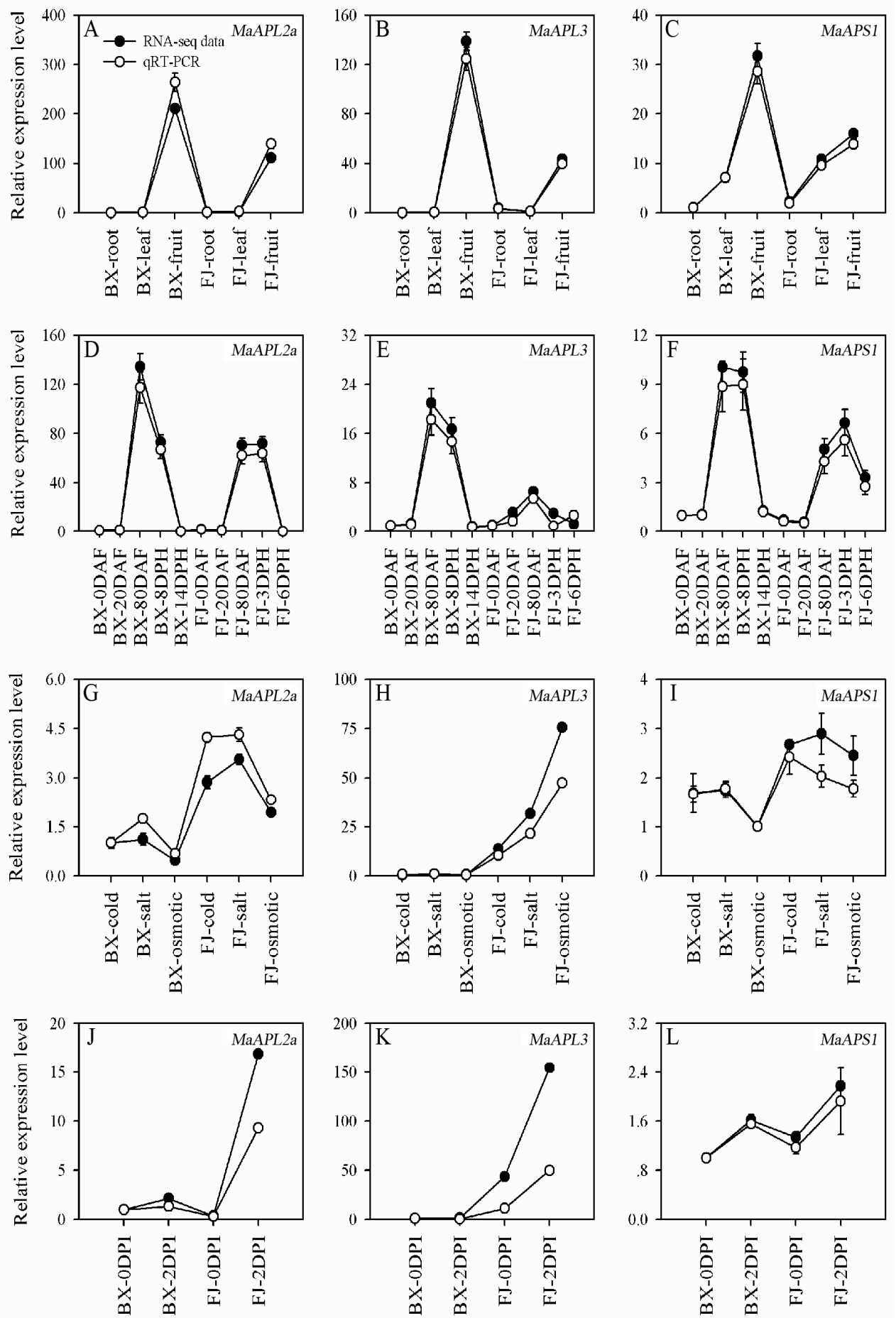

Figure 8. Relative expression of MaAGPases in two banana varieties, BX and FJ, by qRT-PCR. (A-C) Expression of MaAPL-2a, -3, and MaAPS1 in different organs. (D-F) Expression of MaAPL-2a, -3 , and MaAPS1 at different stages of fruit development and ripening. (G-I) Expression of MaAPL-2a, -3 , and MaAPS1 in response to cold, salt, and osmotic stresses. (J-L) Expression of MaAPL-2a, -3 , and MaAPS1 in response to fungal infection. Data are presented as means \pm standard deviations of $n=3$ biological replicates. 


\section{Discussion}

In spite of the economic and social importance of bananas, research on banana plants has generally been slow relative to other crops, especially with respect to fruit development and responsiveness to abiotic/biotic stresses [8]. AGPase is the first rate-limiting enzyme in starch biosynthetic pathways and it has also been reported to play crucial roles in the regulation of plant growth, development, and in response to environmental stresses in many plant species $[12,23,29,30]$. Based on genome-wide search in M. acuminata, we have identified eight MaAGPase genes, which could be classified into two distinct groups based on the size of their subunit and phylogenetic relationship. Such a finding is consistent with the classification of AGPases in other higher plants as represented in A. thaliana and $O$. sativa $[15,16]$. The phylogenetic analyses were further supported by gene structure and conserved motif analyses. The large and small subunits in MaAGPases are distinct in exon-intron organization. It was found that the large subunits harbor 14-15 exons, whereas small subunits contain significantly fewer, only 9-10 exons. Such a structural feature of MaAGPases has also been observed in other plant species, such as Triticum aestivum [38], I. batatas [39], and H. vulgare [40]. Moreover, these two types of subunits also differ in their conserved motifs (Figure 3), as previously observed in T. aestivum [10].

The AGPase family has been reported to participate in the fruit development and ripening process in many plant species, such as S. lycopersicum [22], T. aestivum [41], and Vitis vinifera [29]. In S. lycopersicum, APL1 was found highly expressed in developing fruits, implying regulatory control of AGPase activity and heterotetramer stability [22]. In T. aestivum, AGPase expression was closely related to starch synthesis and degradation in pericarp during caryopsis development [41]. In the current study, we found that more than 63\% MaAGPases were expressed during developmental and ripening stages in both $\mathrm{BX}$ and $\mathrm{FJ}$ varieties. More than $25 \%$ of the expressed AGPase genes were highly expressed (FPKM > 10) (Figure 5), implying that MaAGPases may be extensively involved in the fruit development and ripening in banana. In this study, we have also found that both $M a A P L-3$ and $-2 a$ showed specifically high levels of expression during fruit development stage and early-stage ripening, in contrast to MaAPS1 which displayed constantly high expression at all phases of fruit development and ripening. Interestingly, MaAGPase expression patterns at a certain stage varied greatly between genotypes, for instance, the MaAPL2c was expressed more than ten-fold higher in BX than FJ at the late stage of banana fruit development. The BX genotype, with AAA genome, is known to produce high yield and high quality fruits with long fingers and extended shelf life compared to the FJ genotype with $\mathrm{AAB}$ genome [35]. It is tempting to assume that the increase of MaAGPase expression during fruit development augments starch synthesis ability, thereby enhancing the quality and yield of banana fruit. Such a finding in this study is consistent with previous studies suggesting that the A-genome harbors more genes that are important for banana yield and quality and could be used as a target in breeding programs [8,35]. Bananas are extremely sensitive to abiotic stresses, and can suffer heavy yield and quality penalties at cold, salt, or drought stress conditions [35]. In the present study, 63\% MaAGPases showed transcriptional changes following abiotic stress treatments, including cold, salt, and osmotic stresses (Figure 6). In this report, for the first time in banana, we have demonstrated that MaAGPase genes exhibit extensive and diverse responses to abiotic stresses. This is consistent to previously reported induction of AGPase expression by cold, salt, and drought stresses in other plants, such as S. tuberosum [30], S. lycopersicum [28], and T. aestivum [31]. Of particular interest, comparative transcriptome analysis clearly demonstrated that higher number of MaAGPase genes were significantly up-regulated in FJ than in BX when subjected to cold, salt, and drought treatments (Figure 6). It has been reported that B-genome-containing banana varieties are more resilient to abiotic stresses [42,43]. $\mathrm{FJ}$, with its genome constitution as $\mathrm{AAB}$, has been reported to have more tolerance to abiotic stress in comparison to the banana genotypes containing only A-genome [35].

Banana production could be devastated by fungal disease caused by Foc TR4 [44]. In grapevine, the P. viticola infection was found to directly increase AGPase activity [29]. Similarly, up-regulation of AGPase in leaves leading to the enhanced starch biosynthesis as the result of Huanglongbing (HLB) infection has also been reported in citrus species [45]. In addition, depending on the pathosystems, 
an alteration in starch content in plant tissues was found to be related to the interactions between host plant and biotrophic pathogens [46]. In the current study, the transcriptional up-regulation of MaAGPase genes in response to Foc TR4 infection may suggest their possible role in response to fungal infections in banana. Furthermore, the fact that more MaAGPase genes were found in response to Foc TR4 infection in FJ than BX may suggest the possible involvement of B-genome in protection against biotic stress in banana.

In wheat, the first $400 \mathrm{bp}$ of the $5^{\prime}$ region along with the first intron of $88 \mathrm{bps}$ in APL was found to be important for gene expression and starch accumulation in endosperm [47]. More recently, several cis-acting regulatory elements have been identified in the AGPase promoter, which are involved in the spatial and temporal gene expression and in response to abiotic stresses [10,47-49]. In this study, for the first time in banana, we have identified five hormone-related and seven stress-relevant cis-regulatory elements in the promoters of eight MaAGPase genes. Such a finding supports the genotype-wide transcriptome analysis of AGPase genes, which are likely playing an important role in the regulation of multiple development processes and in response to abiotic/biotic stresses in banana.

\section{Materials and Methods}

\subsection{Plant Materials}

Two banana cultivars, BaXi Jiao (M. acuminata AAA group cv. Cavendish, abbreviated as BX) and Fen Jiao ( $M$. AAB group Fenjiao, abbreviated as FJ), were obtained from the banana plantation at the Chinese Academy of Tropical Agricultural Sciences (Danzhou, Hainan, China), and were selected to perform comparative analyses because of their distinct characteristics. BX is a triploid (AAA) cultivar featured with high yield, high quality, and long-term storage. FJ is also a triploid cultivar, but with a different genotype $(\mathrm{AAB})$, featured by good flavor, rapid ripening, and tolerance to abiotic stresses. Root, leaf, and fruit at 80 DAF were selected for spatial AGPase expression analysis. For temporal expression analysis, developing banana fruits of 0,20 , and $80 \mathrm{DAF}$, representing fruit developmental stages of budding, cutting flower, and harvest stages, respectively, were collected from both banana varieties. BX fruits stored for 0,8 , and $14 \mathrm{DPH}$ and FJ fruits at 0,3 , and $6 \mathrm{DPH}$, representing the three progressive ripening stages based on color of the fruit, including green, yellowish green, and yellow, respectively, have been selected for post-harvest analysis. For salt and osmotic treatments, five-leaf stage banana plants (grown at $28^{\circ} \mathrm{C}, 70 \%$ relative humidity, $200 \mu \mathrm{mol} \cdot \mathrm{m}^{-2} \cdot \mathrm{s}^{-1}$ light intensity, and $16 \mathrm{~h} \mathrm{light} / 8 \mathrm{~h}$ dark cycle) were irrigated with $300 \mathrm{mmol} \cdot \mathrm{L}^{-1} \mathrm{NaCl}$ and $200 \mathrm{mmol} \cdot \mathrm{L}^{-1}$ mannitol for 7 days. For cold treatment, banana plants were maintained at $4{ }^{\circ} \mathrm{C}$ for $22 \mathrm{~h}$ prior to analysis. For Foc TR4 treatments, roots of five-leaf stage banana seedlings were dipped in a Foc TR4 spore suspension of $1.5 \times 10^{6}$ condia $/ \mathrm{mL}$. The entire root system was harvested at 0 and $2 \mathrm{DPI}$, immediately frozen in liquid nitrogen, and stored at $-80^{\circ} \mathrm{C}$ until expression analysis.

\subsection{Identification and Phylogenetic Analyses of MaAGPase Proteins in Banana}

Banana AGPase proteins were downloaded from the DH-Pahang (M. acuminata, A-genome, $2 n=22$ ) genome database (Available online: http://banana-genome.cirad.fr) [34]. AGPase amino acid sequences from $A$. thaliana and O. sativa were obtained from the TAIR (Available online: http: //www.arabidopsis.org) and RGAP (Available online: http:/ / rice.plantbiology.msu.edu) databases, respectively. The typically conserved NTP transferase domains (PF00483, Available online: http: // pfam.sanger.ac.uk) in AGPase were used to query the predicted banana AGPase proteins using HMMER software (Available online: http:/ / hmmer.org). BLAST analysis was also used to identify the predicted banana AGPases with all the AGPases from A. thaliana and O. sativa queries. The conserved domain search of the potential banana AGPases was further validated using conserved domain database (CDD) (Available online: http:/ / www.ncbi.nlm.nih.gov/cdd) and PFAM (Available online: http:/ / pfam.sanger.ac.uk) databases. The accession number of all identified banana AGPases were added in Table S1. A bootstrap neighbor-joining phylogenetic tree with the deduced AGPase amino 
acids sequences from M. acuminata, A. thaliana, and O. sativa were constructed by Clustal X 2.0 and MEGA 5.0 software with bootstrap values for 1000 replicates [50].

\subsection{Characterization Analysis of Protein Properties and Gene Structure}

Molecular mass and isoelectric points of the MaAGPase proteins were predicted by the ExPASy database (Available online: http://expasy.org/). MaAGPase protein motifs were analyzed with MEME software (Available online: http:/ / meme-suite.org) and annotated by searching InterProScan database (Available online: http://www.ebi.ac.uk/Tools/pfa/iprscan). Gene structure features of MaAGPases were identified using GSDS software (Available online: http:/ / gsds.cbi.pku.edu.cn) by comparing genomic sequences and predicted coding regions. Promoter sequences of MaAGPase genes were obtained from the banana genome database (Available online: http:/ /banana-genome.cirad.fr). A fragment of $2000 \mathrm{bp}$ upstream sequences derived from each MaAGPase gene was used to predict the transcription start site using database (Available online: http:/ / www.fruitfly.org/seq_tools/promoter. $\mathrm{html}$ ) and the cis-acting elements via PlantCARE software (Available online: http:/ / bioinformatics. psb.ugent.be/webtools/plantcare/html).

\subsection{Transcriptomic Analysis}

Transcript accumulation of MaAGPase genes in different organs, at different developmental and ripening stages, and under different abiotic/biotic stresses was investigated in the $\mathrm{BX}$ and $\mathrm{FJ}$ genotypes. Roots, leaves, and fruits at $80 \mathrm{DAF}$ were used for the expression analysis of MaAGPase genes in different tissues. Pulps at 0,20, and 80 DAF were collected for the expression analysis of MaAGPase genes during fruit development. Pulps at 8 and $14 \mathrm{DPH}$ in BX and at 3 and $6 \mathrm{DPH}$ in FJ were collected for expression analysis of MaAGPase genes during fruit postharvest ripening. The leaves derived from banana seedlings at five-leaf stage treated with $200 \mathrm{mM}$ mannitol for 7 days, $300 \mathrm{mM} \mathrm{NaCl}$ for 7 days, or low temperature $\left(4^{\circ} \mathrm{C}\right)$, or Foc TR4 treatments were collected to expression analysis of MaAGPase genes under different abiotic/biotic stresses. All samples were collected and frozen quickly in liquid nitrogen and stored at $-80^{\circ} \mathrm{C}$ for RNA extraction. Total RNA was extracted using the plant RNAprep pure Kit (TIANGEN Biotech, Beijing, China) and used to construct cDNA libraries following manufacturer's instruction. Deep sequencing was performed with the GAII kit (Illumina, San Diego, CA, USA) following manufacturer's instructions with two technical replicates per sample. The sequencing depth was $5.34 \times$ on average. Adapter sequences in the raw sequence reads and low quality sequences were removed using FASTX-toolkit and FastQC, respectively, and clean reads were obtained and mapped to the DH-Pahang genome (M. acuminata, $2 n=22$, A genome). Transcriptome assemblies were constructed with Cufflinks [35]. Gene expression levels were calculated as FPKM. DEGseq was used to identify differentially expressed genes.

\subsection{QRT-PCR Analysis}

Expression levels of MaAGPases in different tissues, different stages of fruit development and ripening, and in response to cold, salt, osmotic, and Foc TR4 stresses were further analyzed by qRT-PCR analysis using SYBR ${ }^{\circledR}$ Premix $E x$ Taq $^{\text {TM }}$ kit (TaKaRa, Shiga, Japan) chemistry on a Stratagene Mx3000P detection system (Stratagene, San Diego, CA). Primer pairs that had high specificity and efficiency according to melting curve analysis and agarose gel electrophoresis were selected to conduct quantification analysis (Table S8). Amplification efficiencies of primer pairs ranged from 0.9 to 1.1 . The banana MaActin (EF672732) and MaUBQ2 (HQ853254) were selected as internal controls to normalize the relative expression levels of MaAGPase genes. The relative expression levels of $M a A G P a s e s$ were assessed based on $2^{-\Delta \Delta C t}$ method [51]. Each sample contains three replicates. 


\subsection{Statistical Analysis}

Three biological replicates were performed for each sample. Statistical analyses were performed using Microsoft Excel and SPSS (Chicago, IL). Analysis of variance was used to compare the statistical difference based on Student's $t$-tests, at significant levels of $p<0.05\left(^{*}\right)$ and $p<0.01\left(^{* *}\right)$.

\section{Conclusions}

We identified eight MaAGPase genes from the banana genome, which could be classified into APL and APS, representing the large and small subunits respectively, following phylogenetic analysis, gene structure, and conserved protein motif analyses. Spatial and temporal expression profiles of MaAGPases in two triploid banana varieties revealed that MaAPLs and MaAPSs are differentially expressed during fruit development and ripening, suggesting distinct functions during the fruit development and ripening. The expression patterns of MaAGPases in response to abiotic/biotic stresses may shed light on their possible involvements in stress signaling pathway regulation, especially in the B-genome-containing banana varieties. Furthermore, the identification of hormone-related and stress-related cis-acting elements in MaAGPase promoters provides further evidence for their active role in response to fruit development, ripening, and abiotic/biotic stress signaling. These results will advance our understanding of the functional characterization of AGPase genes in banana, and provide a solid foundation for further genetic improvement of banana fruit quality and resistance to various environmental stresses.

Supplementary Materials: Supplementary materials can be found at www.mdpi.com/1422-0067/18/8/1581/s1.

Acknowledgments: This work was supported by the National Natural Science Foundation of China (NSFC, No.31401843), the Modern Agro-industry Technology Research System (No.CARS-32), the Central Public-interest Scientific Institution Basal Research Fund for Innovative Research Team Program of CATAS (No.1630052017010 and 1630052017018), and the Central Public-interest Scientific Institution Basal Research Fund for Chinese Academy of Tropical Agricultural Sciences (No. 1630052016006).

Author Contributions: Conceived and designed the experiments: Biyu $\mathrm{Xu}$ and Zhiqiang Jin; Performed the experiments: Hongxia Miao and Peiguang Sun; Analyzed the data: Hongxia Miao, Peiguang Sun, and Qing Liu; Contributed reagents/materials/analysis tools: Hongxia Miao, Peiguang Sun, Juhua Liu, Biyu Xu, and Zhiqiang Jin. Wrote the paper: Hongxia Miao, Peiguang Sun, and Qing Liu.

Conflicts of Interest: The authors declare no conflict of interest.

\section{References}

1. Li, Y.; Li, C.; Gu, Z.; Hong, Y.; Cheng, L.; Li, Z. Effect of modification with 1,4- $\alpha$-glucan branching enzyme on the rheological properties of cassava starch. Int. J. Biol. Macromol. 2017, 103, 630-639. [CrossRef] [PubMed]

2. Tiessen, A.; Hendriks, J.H.; Stitt, M.; Branscheid, A.; Gibon, Y.; Farré, E.M.; Geigenberger, P. Starch synthesis in potato tubers is regulated by post-translational redox modification of ADP-glucose pyrophosphorylase: A novel regulatory mechanism linking starch synthesis to the sucrose supply. Plant Cell 2002, 14, 2191-2213. [CrossRef] [PubMed]

3. Toyosawa, Y.; Kawagoe, Y.; Matsushima, R.; Crofts, N.; Ogawa, M.; Fukuda, M.; Kumamaru, T.; Okazaki, Y.; Kusano, M.; Saito, K.; et al. Deficiency of starch synthase IIIa and IVb alters starch granule morphology from polyhedral to spherical in rice endosperm. Plant Physiol. 2016, 170, 1255-1270. [CrossRef] [PubMed]

4. Zeeman, S.C.; Kossmann, J.; Smith, A.M. Starch: Its metabolism, evolution, and biotechnological modification in plants. Annu. Rev. Plant Biol. 2010, 61, 209-234. [CrossRef] [PubMed]

5. Jourda, C.; Cardi, C.; Gibert, O.; Giraldo Toro, A.; Ricci, J.; Mbéguié-A-Mbéguié, D.; Yahiaoui, N. Lineage-specific evolutionary histories and regulation of major starch metabolism genes during banana ripening. Front. Plant Sci. 2016, 7, 1778. [CrossRef] [PubMed]

6. Miao, H.X.; Sun, P.G.; Liu, W.X.; Xu, B.Y.; Jin, Z.Q. Identification of genes encoding granule-bound starch synthase involved in amylose metabolism in banana fruit. PLoS ONE 2014, 9, e88077. [CrossRef] [PubMed]

7. Miao, H.X.; Sun, P.G.; Liu, Q.; Jia, C.H.; Liu, J.H.; Hu, W.; Jin, Z.Q.; Xu, B.Y. Soluble starch synthase III-1 in amylopectin metabolism of banana fruit: Characterization, expression, enzyme activity, and functional analyses. Front. Plant Sci. 2017, 8, 454. [CrossRef] [PubMed] 
8. Miao, H.X.; Sun, P.G.; Liu, Q.; Miao, Y.L.; Liu, J.H.; Zhang, K.X.; Hu, W.; Zhang, J.B.; Wang, J.Y.; Wang, Z.; et al. Genome-wide analyses of SWEET family proteins reveal involvement in fruit development and abiotic/biotic stress responses in banana. Sci. Rep. 2017, 7, 3536. [CrossRef] [PubMed]

9. Smith, A.M.; Denyer, K.; Martin, C. The synthesis of the starch granule. Annu. Rev. Plant Physiol. Plant Mol. Biol. 1997, 48, 67-87. [CrossRef] [PubMed]

10. Batra, R.; Saripalli, G.; Mohan, A.; Gupta, S.; Gill, K.S.; Varadwaj, P.K.; Balyan, H.S.; Gupta, P.K. Comparative analysis of AGPase genes and encoded proteins in eight monocots and three dicots with emphasis on wheat. Front. Plant Sci. 2017, 8, 19. [CrossRef] [PubMed]

11. Martin, C.; Smith, A.M. Starch biosynthesis. Plant Cell 1995, 7, 971-985. [CrossRef] [PubMed]

12. Saripalli, G.; Gupta, P.K. AGPase: Its role in crop productivity with emphasis on heat tolerance in cereals. Theor. Appl. Genet. 2015, 128, 1893-1916. [CrossRef] [PubMed]

13. Hannah, L.C.; Shaw, J.R.; Giroux, M.J.; Reyss, A.; Prioul, J.L.; Bae, J.M.; Lee, J.Y. Maize genes encoding the small subunit of ADP-glucose pyrophosphorylase. Plant Physiol. 2001, 127, 173-183. [CrossRef] [PubMed]

14. Tiessen, A.; Nerlich, A.; Faix, B.; Hümmer, C.; Fox, S.; Trafford, K.; Weber, H.; Weschke, W.; Geigenberger, P. Subcelluar analysis of starch metabolism in developing barley seeds using a non-aqueous fractionation method. J. Exp. Bot. 2012, 63, 2071-2087. [CrossRef] [PubMed]

15. Crevillén, P.; Ventriglia, T.; Pinto, F.; Orea, A.; Mérida, Á.; Romero, J.M. Differential pattern of expression and sugar regulation of Arabidopsis thaliana ADP-glucose pyrophosphorylase-encoding genes. J. Biol. Chem. 2005, 280, 8143-8149. [CrossRef] [PubMed]

16. Lu, F.H.; Park, Y.J. Sequence variations in OsAGPase significantly associated with amylose content and viscosity properties in rice (Oryza sativa L.). Genet. Res. 2012, 94, 179-189. [CrossRef] [PubMed]

17. Tang, X.J.; Peng, C.; Zhang, J.; Cai, Y.; You, X.M.; Kong, F.; Yan, H.G.; Wang, G.X.; Wang, L.; Jin, J.; et al. ADP-glucose pyrophosphorylase large subunit 2 is essential for storage substance accumulation and subunit interactions in rice endosperm. Plant Sci. 2016, 249, 70-83. [CrossRef] [PubMed]

18. Huang, B.; Hennen-Bierwagen, T.A.; Myers, A.M. Functions of multiple genes encoding ADP-glucose pyrophosphorylase subunits in maize endosperm, embryo, and leaf. Plant Physiol. 2014, 164, 596-611. [CrossRef] [PubMed]

19. Zhou, Y.X.; Chen, Y.X.; Tao, X.; Cheng, X.J.; Wang, H.Y. Isolation and characterization of cDNAs and genomic DNAs encoding ADP-glucose pyrophosphorylase large and small subunits from sweet potato. Mol. Genet. Genom. 2016, 291, 609-620. [CrossRef] [PubMed]

20. Au, S.L.; Tan, S.H.; Harikrishna, K.; Napis, S. Isolation and characterization of cDNA clones encoding ADP-glucose pyrophosphorylase from sago palm (Metroxylon sagu). J. Biochem. Mol. Biol. Biophys. 2002, 6, 301-308. [CrossRef] [PubMed]

21. Nardozza, S.; Boldingh, H.L.; Osorio, S.; Höhne, M.; Wohlers, M.; Gleave, A.P.; MacRae, E.A.; Richardson, A.C.; Atkinson, R.G.; Sulpice, R.; et al. Metabolic analysis of kiwifruit (Actinidia deliciosa) berries from extreme genotypes reveals hallmarks for fruit starch metabolism. J. Exp. Bot. 2013, 64, 5049-5063. [CrossRef] [PubMed]

22. Petreikov, M.; Shen, S.; Yeselson, Y.; Levin, I.; Bar, M.; Schaffer, A.A. Temporally extended gene expression of the ADP-Glc pyrophosphorylase large subunit (AgpL1) leads to increased enzyme activity in developing tomato fruit. Planta 2006, 224, 1465-1479. [CrossRef] [PubMed]

23. Mugford, S.T.; Fernandez, O.; Brinton, J.; Flis, A.; Krohn, N.; Encke, B.; Feil, R.; Sulpice, R.; Lunn, J.E.; Stitt, M.; et al. Regulatory properties of ADP glucose pyrophosphorylase are required for adjustment of leaf starch synthesis in different photoperiods. Plant Physiol. 2014, 166, 1733-1747. [CrossRef] [PubMed]

24. Sulmon, C.; Gouesbet, G.; Ramel, F.; Cabello-Hurtado, F.; Penno, C.; Bechtold, N.; Couée, I.; El Amrani, A. Carbon dynamics, development and stress responses in Arabidopsis: Involvement of the APL4 subunit of ADP-glucose pyrophosphorylase (starch synthesis). PLoS ONE 2011, 6, e26855. [CrossRef] [PubMed]

25. Kwak, M.S.; Min, S.R.; Lee, S.M.; Kim, K.N.; Liu, J.R.; Paek, K.H.; Shin, J.S.; Bae, J.M. A sepal-expressed ADP-glucose pyrophosphorylase gene (NtAGP) is required for petal expansion growth in 'Xanthi' tobacco. Plant Physiol. 2007, 145, 277-289. [CrossRef] [PubMed]

26. Taliercio, E. Characterization of an ADP-glucose pyrophosphorylase small subunit gene expressed in developing cotton (Gossypium hirsutum) fibers. Mol. Biol. Rep. 2011, 38, 2967-2973. [CrossRef] [PubMed] 
27. Lee, S.K.; Eom, J.S.; Hwang, S.K.; Shin, D.; An, G.; Okita, T.W.; Jeon, J.S. Plastidic phosphoglucomutase and ADP-glucose pyrophosphorylase mutants impair starch synthesis in rice pollen grains and cause male sterility. J. Exp. Bot. 2016, 67, 5557-5569. [CrossRef] [PubMed]

28. Yin, Y.G.; Kobayashi, Y.; Sanuki, A.; Kondo, S.; Fukuda, N.; Ezura, H.; Sugaya, S.; Matsukura, C. Salinity induces carbohydrate accumulation and sugar-regulated starch biosynthetic genes in tomato (Solanum lycopersicum L. cv. 'Micro-Tom') fruits in an ABA- and osmotic stress-independent manner. J. Exp. Bot. 2010, 61, 563-574. [CrossRef] [PubMed]

29. Gamm, M.; Héloir, M.C.; Bligny, R.; Vaillant-Gaveau, N.; Trouvelot, S.; Alcaraz, G.; Frettinger, P.; Clément, C.; Pugin, A.; Wendehenne, D.; et al. Changes in carbohydrate metabolism in Plasmopara viticola-infected grapevine leaves. Mol. Plant Microbe Interact. 2011, 24, 1061-1073. [CrossRef] [PubMed]

30. Wiberley-Bradford, A.E.; Busse, J.S.; Jiang, J.; Bethke, P.C. Sugar metabolism, chip color, invertase activity, and gene expression during long-term cold storage of potato (Solanum tuberosum) tubers from wild-type and vacuolar invertase silencing lines of Katahdin. BMC Res. Notes 2014, 7, 801. [CrossRef] [PubMed]

31. Majoul-Haddad, T.; Bancel, E.; Martre, P.; Triboi, E.; Branlard, G. Effect of short heat shocks applied during grain development on wheat (Triticum aestivum L.) grain proteome. J. Cereal Sci. 2013, 57, 486-495. [CrossRef]

32. Thitisaksakul, M.; Jimenez, R.C.; Arias, M.C.; Beckles, D.M. Effects of environmental factors on cereal starch biosynthesis and composition. J. Cereal Sci. 2012, 56, 67-80. [CrossRef]

33. Gibert, O.; Dufour, D.; Giraldo, A.; Sánchez, T.; Reynes, M.; Pain, J.P.; González, A.; Fernández, A.; Díaz, A. Differentiation between cooking bananas and dessert bananas. 1. Morphological and compositional characterization of cultivated Colombian Musaceae (Musa sp.) in relation to consumer preferences. J. Agric. Food Chem. 2009, 57, 7857-7869. [CrossRef] [PubMed]

34. D’Hont, A.; Denoeud, F.; Aury, J.M.; Baurens, F.C.; Carreel, F.; Garsmeur, O.; Noel, B.; Bocs, S.; Droc, G.; Rouard, M.; et al. The banana (Musa acuminata) genome and the evolution of monocotyledonous plants. Nature 2012, 488, 213-217. [CrossRef] [PubMed]

35. Hu, W.; Wang, L.; Tie, W.; Yan, Y.; Ding, Z.; Liu, J.; Li, M.; Peng, M.; Xu, B.; Jin, Z. Genome-wide analyses of the bZIP family reveal their involvement in the development, ripening and abiotic stress response in banana. Sci. Rep. 2016, 6, 30203. [CrossRef] [PubMed]

36. Asif, M.H.; Lakhwani, D.; Pathak, S.; Bhambhani, S.; Baq, S.K.; Trivedi, P.K. Genome-wide identification and expression analysis of the mitogen-activated protein kinase gene family from banana suggest involvement of specific members in different stages of fruit ripening. Funct. Integr. Genom. 2014, 14, 161-175. [CrossRef] [PubMed]

37. Backiyarani, S.; Uma, S.; Nithya, S.; Chandrasekar, A.; Saraswathi, M.S.; Thanqavelu, R.; Mayilvaqanan, M.; Sundararaju, P.; Sinqh, N.K. Genome-wide analysis and differential expression of chitinases in banana against root lesion nematode (Pratylenchus coffeae) and eumusa leaf spot (Mycosphaerella eumusae) pathogens. Appl. Biochem. Biotechnol. 2015, 175, 3585-3598. [CrossRef] [PubMed]

38. Rose, M.K.; Huang, X.Q.; Brûlé-Babel, A. Molecular characterization and sequence diversity of genes encoding the large subunit of the ADP-glucose pyrophosphorylase in wheat (Triticum aestivum L.). J. Appl. Genet. 2016, 57, 15-25. [CrossRef] [PubMed]

39. Noh, S.A.; Kwak, M.S.; Lee, H.S.; Huh, G.H.; Liu, J.R.; Shin, J.S.; Bae, J.M. Genomic organizations of two small subunit ADP-glucose pyrophosphorylase genes from sweet potato. Gene 2004, 339, 173-180. [CrossRef] [PubMed]

40. Thorbjørnsen, T.; Villand, P.; Kleczkowski, L.A.; Olsen, O.A. A single gene encodes two different transcripts for the ADP-glucose pyrophosphorylase small subunit from barley (Hordeum vulgare). Biochem. J. 1996, 313, 149-154. [CrossRef] [PubMed]

41. Yu, X.; Li, B.; Wang, L.; Chen, X.; Wang, W.; Wang, Z.; Xiong, F. Systematic analysis of pericarp starch accumulation and degradation during wheat caryopsis development. PLoS ONE 2015, 10, e0138228. [CrossRef] [PubMed]

42. Tripathi, L.; Odipio, J.; Tripathi, J.N.; Tusiime, G. A rapid technique for screening banana cultivars for resistance to Xanthomonas wilt. Eur. J. Plant Pathol. 2008, 121, 9-19. [CrossRef]

43. Vanhove, A.C.; Vermaelen, W.; Panis, B.; Swennen, R.; Carpentier, S.C. Screening the banana biodiversity for drought tolerance: Can an in vitro growth model and proteomics be used as a tool to discover tolerant varieties and understand homeostasis. Front. Plant Sci. 2012, 3, 176. [CrossRef] [PubMed] 
44. Wang, Z.; Zhang, J.; Jia, C.; Liu, J.; Li, Y.; Yin, X.; Xu, B.; Jin, Z. De novo characterization of the banana root transcriptome and analysis of gene expression under Fusarium oxysporum f. sp. Cubense tropical race 4 infection. BMC Genom. 2012, 13, 650. [CrossRef] [PubMed]

45. Kim, J.S.; Sagaram, U.S.; Burns, J.K.; Li, J.L.; Wang, N. Response of sweet orange (Citrus sinensis) to 'Candidatus Liberibacter asiaticus' infection: Microscopy and microarray analyses. Phytopathology 2009, 99, 50-57. [CrossRef] [PubMed]

46. Horst, R.J.; Engelsdorf, T.; Sonnewald, U.; Voll, L.M. Infection of maize leaves with Ustilago maydis prevents establishment of C4 photosynthesis. J. Plant Physiol. 2008, 165, 19-28. [CrossRef] [PubMed]

47. Thorneycroft, D.; Hosein, F.; Thangavelu, M.; Clark, J.; Vizir, L.; Burrell, M.M.; Ainsworth, C. Characterization of a gene from chromosome 1B encoding the large subunit of ADP glucose pyrophorylase from wheat: Evolutionary divergence and differential expression of Agp2 genes between leaves and developing endosperm. Plant Biotechnol. J. 2003, 1, 259-270. [CrossRef] [PubMed]

48. Liu, G.; Wu, Y.; Xu, M.; Gao, T.; Wang, P.; Wang, L.; Guo, T.; Kang, G. Virus-induced gene silencing identifies an important role of the TaRSR1 transcription factor in starch synthesis in bread wheat. Int. J. Mol. Sci. 2016, 17, 1557. [CrossRef] [PubMed]

49. Hu, X.; Kuang, S.; Zhang, A.D.; Zhang, W.S.; Chen, M.J.; Yin, X.R.; Chen, K.S. Characterization of starch degradation related genes in postharvest kiwifruit. Int. J. Mol. Sci. 2016, 17, 2112. [CrossRef] [PubMed]

50. Li, P.; Miao, H.X.; Ma, Y.W.; Wang, L.; Hu, G.B.; Ye, Z.X.; Zhao, J.T.; Qin, Y.H. CrSKP1, an SKP1-like gene, is involved in the self-incompatibility reaction of "Wuzishatangju" (Citrus reticulata Blanco). Int. J. Mol. Sci. 2015, 16, 21695-21710. [CrossRef] [PubMed]

51. Livak, K.J.; Schmittgen, T.D. Analysis of relative gene expression data using real-time quantitative PCR and the $2^{-\Delta \Delta C t}$ Method. Methods 2001, 25, 402-408. [CrossRef] [PubMed]

(C) 2017 by the authors. Licensee MDPI, Basel, Switzerland. This article is an open access article distributed under the terms and conditions of the Creative Commons Attribution (CC BY) license (http://creativecommons.org/licenses/by/4.0/). 